\title{
Medium modifications of vector mesons
}

\section{György Wolf*}

KFKI RMKI H-1525 Budapest POB. 49, Hungary

E-mail: 'wolf@rmki.kfki . hü

\section{Matthias F.M. Lutz}

GSI, D-64220 Darmstadt, Germany, and

Institut für Kernphysik, TU Darmstadt

D-64289 Darmstadt, Germany

E-mail: 'm. lutz@gsi deí

ABSTRACT: We construct a relativistic and unitary approach to pion- and photon-nucleon scattering taking into account the inelastic $\pi \Delta, \rho N, \omega N, \eta N, K \Lambda, K \Sigma$ channels. Our scheme dynamically generates the s- and d-wave nucleon resonances $N(1535), N(1650)$, $N(1520)$ and $N(1700)$ and isobar resonances $\Delta(1620)$ and $\Delta(1700)$ in terms of quasilocal two-body interaction terms. We obtain a fair description of the experimental data relevant for slow vector-meson propagation in nuclear matter. The s-wave $\rho$ - and $\omega$-meson nucleon scattering amplitudes, which define the leading density modification of the $\rho$ - and $\omega$-meson spectral functions in nuclear matter, are predicted.

\section{Introduction}

The decay of vector mesons into $e^{+} e^{-}$and $\mu^{+} \mu^{-}$pairs offers a unique tool to explore the properties of dense and hot matter in nuclear collisions. The lepton pairs provide virtually undistorted information on the current-current correlation function $\left\langle j_{\mu} j_{\nu}\right\rangle$ in the medium [i]. At invariant masses in the range $500-800 \mathrm{MeV},\left\langle j_{\mu} j_{\nu}\right\rangle$ is sensitive to inmedium modifications of the mass distribution of the light vector mesons $\rho$ and $\omega$. Indeed, the observed enhancement of the di-lepton yield at small invariant masses [i2] is presently interpreted in terms of in-medium modifications of the mass and width of the $\rho$ meson

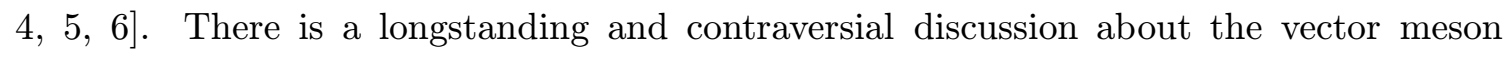
properties in dense nuclear matter. To leading orders in the baryon density, modifications of the mass distribution are determined by the vector-meson nucleon scattering amplitudes. Since these amplitudes are not directly constrained by data, the predictions for the vectormeson spectral densities in nuclear matter are strongly model dependent.

\footnotetext{
${ }^{*}$ Speaker.
} 
This work is an attempt to overcome this problem by using the constraints from data on pion- and photon-nucleon scattering considering in particular the $\omega$ - and $\rho$-meson production data in a systematic way. We construct a coupled-channel scheme for meson-baryon scattering, including the $\pi N, \rho N, \omega N, \pi \Delta, \eta N, K \Lambda$ and $K \Sigma$ channels. In such a scheme the amplitudes for experimentally non-accessible processes like $\rho N$ and $\omega N$ scattering are constrained by the data on elastic $\pi N$ scattering and inelastic reactions like the pion- and photon-induced production of vector mesons. Our goal is to determine the vector-meson nucleon scattering amplitudes close to threshold. Consequently we concentrate on the energy window $1.4 \mathrm{GeV}<\sqrt{s}<1.8 \mathrm{GeV}$. It is sufficient to consider only s-wave scattering in the $\rho N$ and $\omega N$ channels. This implies that in the $\pi N$ and $\pi \Delta$ channels we need only sand d-waves. In particular, we consider the $S_{11}, S_{31}, D_{13}$ and $D_{33}$ partial waves of $\pi N$ scattering. In order to systematically derive the momentum dependence of the vector-meson self energy, vector-meson nucleon scattering also in higher partial waves would have to be considered.

\section{Relativistic coupled-channel dynamics}

Here we only outline our model, the detailed discusion can be found in $\left[\overline{\bar{T}_{1}}\right]$. We construct an effective Lagrangian with quasi-local four-point meson-baryon contact interactions in order to study the meson-baryon scattering process. Within this framework, the Bethe-Salpeter equation for the coupled-channel system reduces to a matrix equation.

The general four-point interaction Lagrangian for the coupled-channel problem in momentum space can be written in the form

$$
\mathcal{L}(\bar{k}, k ; w)=\sum_{I} R^{(I) \dagger}(\bar{q}, \bar{p}) \gamma_{0} K^{(I)}(\bar{k}, k ; w) R^{(I)}(q, p)+\ldots,
$$

where $K_{a b}^{(I)}(\bar{k}, k ; w)$ is a channel dependent interaction matrix, which in general is a function of the relative momenta of the initial and final states $k=\frac{1}{2}(p-q)$ and $\bar{k}=\frac{1}{2}(\bar{p}-\bar{q})$ as well as the total momentum $w=p+q=\bar{p}+\bar{q}$. The state vectors $R^{(I)}(q, p)$ involve the positive frequency parts of the fields specifying the various channels.

The coupled-channel Bethe-Salpeter equation projected onto a given isospin then reads

$$
\begin{aligned}
T_{a b}^{(I)}(\bar{k}, k ; w) & =K_{a b}^{(I)}(\bar{k}, k ; w)+\sum_{c, d} \int \frac{d^{4} l}{(2 \pi)^{4}} K_{a c}^{(I)}(\bar{k}, l ; w) G_{c d}^{(I)}(l ; w) T_{d b}^{(I)}(l, k ; w), \\
G_{c d}^{(I)}(l ; w) & =-i D_{\Phi(I, c) \Phi(I, d)}\left(\frac{1}{2} w-l\right) S_{B(I, c) B(I, d)}\left(\frac{1}{2} w+l\right),
\end{aligned}
$$

where $D_{\Phi(I, c) \Phi(I, d)}(q)$ and $S_{B(I, c) B(I, d)}(p)$ denote the meson and baryon Feynman propagators in a given channel of isospin $I$. A convenient labelling of the channels is obtained by defining

$$
\begin{array}{ll}
\Phi\left(\frac{1}{2}, a\right)=\left(\pi, \pi, \rho_{\mu}, \omega_{\mu}, \eta, K, K\right)_{a}, & B\left(\frac{1}{2}, a\right)=\left(N, \Delta_{\mu}, N, N, N, \Lambda, \Sigma\right)_{a}, \\
\Phi\left(\frac{3}{2}, a\right)=\left(\pi, \pi, \rho_{\mu}, K\right)_{a}, & B\left(\frac{3}{2}, a\right)=\left(N, \Delta_{\mu}, N, \Sigma\right)_{a} .
\end{array}
$$


The general form of the interaction kernel may be obtained from a meson exchange model or more systematically from the chiral Lagrangian. In this paper we construct an effective field theory for meson-nucleon scattering in the resonance region. The philosophy of this approach is to approximate the interaction kernel $K$ but to treat rescattering in the s-channel explicitly. In our scheme, we assume that the interaction kernel is slowly varying in energy in the relevant window $1.4 \mathrm{GeV}<\sqrt{s}<1.8 \mathrm{GeV}$.

We constrain our model parameters by data from photon-induced meson production off the nucleon. According to the original conjecture of Sakurai [is] the vector-meson converts into a real photon via the interaction terms $A^{\mu} \rho_{\mu}^{(0)}$ and $A^{\mu} \omega_{\mu}$. In our scheme these terms are not allowed. In order to simulate the successful vector-meson dominance assumption it is therefore natural to directly relate the strength of the $\gamma N \rightarrow X$ and $\rho^{(0)} N \rightarrow X$, $\omega N \rightarrow X$ vertices, where $X$ is any hadronic final state. Note that our construction has similarities with the construction of Kroll, Lee and Zumino [9. from insisting that the vector mesons couple to conserved currents only.

We specify our generalized form of the vector-meson dominance conjecture as applied for the direct photon-induced production vertices $K_{\gamma N \rightarrow X}^{(I), \mu}(\bar{q}, q ; w)$ :

$$
\begin{aligned}
& \frac{1}{2}\left(K_{\gamma p \rightarrow X}^{\left(\frac{1}{2}\right), \mu}+K_{\gamma n \rightarrow X}^{\left(\frac{1}{2}\right), \mu}\right)(\bar{q}, q ; w)=e K_{\nu, \omega N \rightarrow X}^{\left(\frac{1}{2}\right)}(\bar{q}, q ; w) \Gamma_{S}^{\nu \mu}(q ; w), \\
& \frac{1}{2}\left(K_{\gamma p \rightarrow X}^{\left(\frac{1}{2}\right), \mu}-K_{\gamma n \rightarrow X}^{\left(\frac{1}{2}\right), \mu}\right)(\bar{q}, q ; w)=\frac{e}{\sqrt{3}} K_{\nu, \rho N \rightarrow X}^{\left(\frac{1}{2}\right)}(\bar{q}, q ; w) \Gamma_{V}^{\nu \mu}(q ; w), \\
& K_{\gamma p \rightarrow X}^{\left(\frac{3}{2}\right), \mu}(\bar{q}, q ; w)=e \sqrt{\frac{2}{3}} K_{\nu, \rho N \rightarrow X}^{\left(\frac{3}{2}\right)}(\bar{q}, q ; w) \Gamma_{V}^{\nu \mu}(q ; w), \\
& K_{\gamma n \rightarrow X}^{\left(\frac{3}{2}\right), \mu}(\bar{q}, q ; w)=e \sqrt{\frac{2}{3}} K_{\nu, \rho N \rightarrow X}^{\left(\frac{3}{2}\right)}(\bar{q}, q ; w) \Gamma_{V}^{\nu \mu}(q ; w),
\end{aligned}
$$

where $X$ stands for any hadronic two-body final state with isospin $I$. The transverse objects $\Gamma_{S(V)}^{\mu \nu}(q ; w)$ are the most general transition tensors compatible with gauge invariance. Its form can be found in [i]i].

\section{Results}

In accordance with the effective field theory approach only data in an appropriate kinematical window is used in the analysis. The threshold for vector-meson production off a nucleon is at $\sqrt{s} \simeq 1.7 \mathrm{GeV}$. Close to the elastic pion-nucleon threshold we do not expect our scheme to be efficient. We therefore fit the data in the energy range $1.4 \mathrm{GeV}$ $\leq \sqrt{s} \leq 1.8 \mathrm{GeV}$, with the energy independent coupling matrices. Our model includes four coupled-channel matrices carrying quantum numbers $I=\frac{1}{2}, \frac{3}{2}$ and $J=\frac{1}{2}, \frac{3}{2}$. As a result of our fit the s- and d-wave nucleon resonances $N(1535), N(1650), N(1520)$ and $N(1700)$ and isobar resonances $\Delta(1620)$ and $\Delta(1700)$ are generated dynamically in our scheme.

The set of parameters is adjusted to describe the partial-wave pion-nucleon phase shifts including their inelasticity parameters. Furthermore the pion- and photon-induced production cross sections of pseudo-scalar meson and vector mesons are considered. Of crucial importance to our fit are the empirical pion- and photon-induced vector-meson production data. Only via the existence of those data it is ultimately possible to determine 
the vector-meson nucleon scattering amplitudes, the prime goal of this work. All together we include $N=1156$ data points. We obtained a fair description of the experimental data [1]기.

We apply the resulting vector-meson scattering amplitudes to the propagation of the $\rho$ and $\omega$ mesons in nuclear matter. To leading order in density the low-density theorem predicts the self energy of a vector meson in nuclear matter in terms of the s-wave scattering amplitude averaged over spin and isospin [i $\left[\mathbf{1}^{i}\right]$.

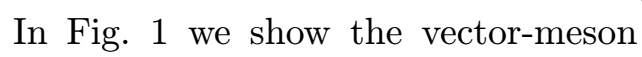
propagators at the saturation density of nuclear matter, $\rho_{0}=0.17 \mathrm{fm}^{-3}$ and at $\rho=$ $2 \rho_{0}$. For the $\rho$ meson we note an enhancement of the width, and a downward shift in energy, due to the mixing with the baryon resonances at $\sqrt{s}=1.5-1.6 \mathrm{GeV}$. At $\rho=\rho_{0}$ the center-of-gravity of the spectral function is shifted down in energy by about $3 \%$. The in-medium propagator of the $\omega$ meson exhibits two distinct quasiparticles, an $\omega$ like mode, which is shifted up somewhat in energy, and a resonance-hole like mode at low energies. The low-lying
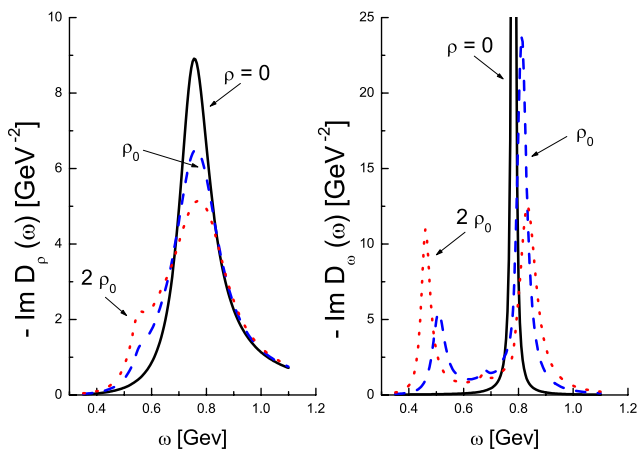

Figure 1: Imaginary parts of the $\rho$ and $\omega$ propagators in nuclear matter at $\rho=\rho_{0}$ and $2 \rho_{0}$, compared to those of the free-space propagators. modes carry about $15 \%$ on the energyweighted sum rule. The center-of-gravity is shifted down by about $4 \%$. However, we stress that the structure of the in-medium $\omega$-meson spectral function clearly cannot be characterized by this number alone.

A simple estimate on the accuracy of the leading order result follows by investigating the size of the leading correction term determined by the Pauli blocking effect. Take for example the $\omega$ meson for which its leading and subleading mass shift terms require only the s-wave scattering length $a_{\omega N}^{\frac{1}{2}}=(-0.45+i 0.31)$ fm and $a_{\omega N}^{\frac{3}{2}}=(-0.43+i 0.15) \mathrm{fm}$ [i] At nuclear saturation density the correction term of order $k_{F}^{4}$ implies a further repulsive mass shift of $1 \mathrm{MeV}$ and increase of the decay width of $2 \mathrm{MeV}$ for the $\omega$ meson. We expect that the results obtained with only the leading term in the low-density expansion are qualitatively correct at normal nuclear matter density. However, on a quantitative level, the spectral functions may change when higher order terms in the density expansion are included. For instance, we expect that the in-medium properties of the baryon resonances depend sensitively on the meson spectral functions. If this is the case, a self consistent calculation, which corresponds to a partial summation of terms in the density expansion, would have to be performed [i] $\left.i_{1}\right]$.

\section{Summary and conclusion}

In this work we predicted the amplitudes which describe the s-wave scattering of the light vector mesons off nucleons. To leading order in a density expansion these amplitudes de- 
termine the spectral functions of the $\rho$ - and $\omega$-meson in nuclear matter. Since there exist no data on vector-meson nucleon scattering we constrained our analysis by all relevant elastic and inelastic $\gamma N$ and $\pi N$ data. The coupled channel unitarity condition together with the causality property of local quantum field theory then leads to quite robust predictions for the vector-meson nucleon scattering amplitudes. Our amplitudes show rapid energy variations due to the presence of nucleon and isobar resonances. In our novel and covariant coupled channel approach, which considers the $\gamma N, \pi N, \pi \Delta, \rho N, \omega N, \eta N, K \Lambda$ and $K \Sigma$ states, the s- and d-wave nucleon and isobar resonances are generated dynamically by coupled channel effects. In nuclear matter our scattering amplitudes lead to a $\omega$-meson spectral functions with considerable support at energies smaller than the free-space mass representing resonance nucleon-hole type excitations. On the other hand, as an immediate consequence of the only moderate coupling of the $\rho N$ channel to the $N(1520) \mathrm{d}$-wave resonance, the in-medium effects for the $\rho$ meson are found to be significantly smaller as compared to previous works.

The results of our work are relevant for the experimental program at GSI. The HADES detector will help to further explore the properties of the light vector mesons in nuclear matter by measuring their dilepton final state with high accuracy. Complementary experimental programs are pursued at MAMI and KEK [1] $\left.{ }_{1}^{1}\right]$ with photon and nucleon induced reactions off nuclei. To further substantiate the structure of the vector-meson nucleon scattering amplitudes it would be desirable to establish a more microscopic understanding of the effective interaction vertices employed in our work.

This work were partly supported by Hungarian Research Foundation (OTKA) grants: T 26543 and T 30855.

\section{References}

[1] R. Rapp and J. Wambach,

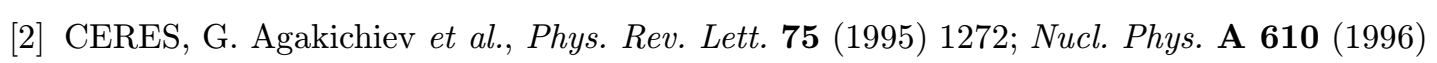
.

[3] G.E. Brown and M. Rho, i Phys. Rev. Lett. 66 $199 \overline{1}) \overline{2} \overline{7} \overline{0}$.

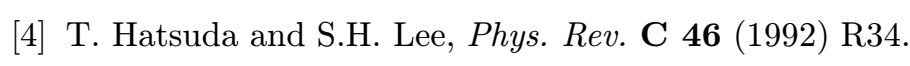

[5] M. Herrmann, B. Friman and W. Nörenberg, iNucl. P $\bar{h} y s$. A $5600(199 \overline{3}) \overline{4} 1 \overline{1}$.

[6] G. Chanfray, R. Rapp and J. Wambach, 'Phys. Rev. Lett. 76 19996$) 368$

[7] M.F.M. Lutz, Gy. Wolf, B. Friman, in preparation.

[8] J.J. Sakurai, Currents and Mesons (University of Chicago Press, Chicago, 1969).

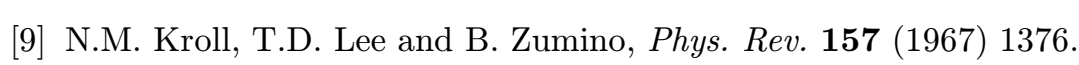

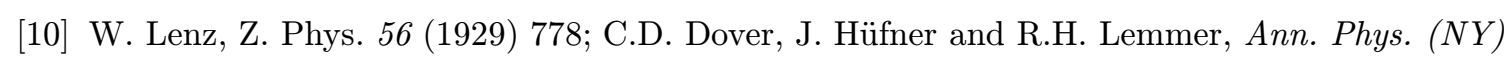
:

[11] M.F.M. Lutz and C. Korpa, nucl-th/0105067.

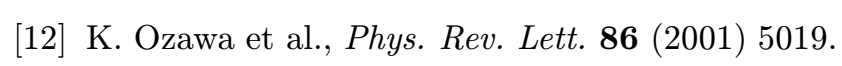

